\title{
On the closure of an elementary abelian 2-subgroup of a finite group
}

\author{
Makoto HAYASHI
}

(Received May 12, 2000)

\begin{abstract}
Let $H$ be a finite group and $S \in \operatorname{Syl}_{2}(H)$. Let $V$ be a $G F(2) H$ module and $U$ be an $S$ submodule of $V$. For an integer $t$, we write $U \in \mu_{t}(S)$ if $U=\left\langle u ; \operatorname{dim}_{G F(2)}[u, A] \leq\right.$ $t)$ for any elementary abelian subgroup $A$ of $S$ with $[U, A, A]=0$. The purpose of this note is to give an estimation of $m$ with $U^{H} \in \mu_{t+m}(S)$, whenever $U \in \mu_{t}(S)$.
\end{abstract}

Key words: the closure of an elementary abelian 2-subgroup, solvable group, component.

Notation All groups considered in this note will be finite. Let $A$ and $B$ be subgroups of a group $H$. We write $A \leq B(A<B)$ to indicate $A$ is a (proper) subgroup of $B$. For a subset $X$ of $H,\langle X\rangle$ denotes the subgroup of $H$ generated by $X .|H|$ denotes the number of elements of $H$, and $H^{\sharp}$ denotes the set of all the nonidentity elements of $H$. Let $a^{b}=b^{-1} a b$ for $a \in A$ and $b \in B, A^{B}=\left\langle a^{b} ; a \in A, b \in B\right\rangle$, and $[A, B]=\left\langle a^{-1} a^{b} ; a \in A, b \in\right.$ $B\rangle . \quad N_{H}(A)$ and $C_{H}(A)$ denote the normalizer and centralizer of $A$ in $H$ respectedly. Let $Z(H)=C_{H}(H)$. For a prime $p, \operatorname{Syl}_{p}(H)$ denotes the set of all Sylow $p$-subgroups of $H, O_{p}(H)$ denotes the largest normal $p$-subgroup of $H$, and $O^{p}(H)$ denotes the subgroup which is generated by all the elements of $H$ whose orders are relatively prime to $p$. $A$ is a component of $H$ if $A$ is a subnormal subgroup of $H$ such that $[A, A]=A$ and $A / Z(A)$ is simple. $E(H)$ denotes the subgroup of $H$ generated by all the components of $H$. Let $X=\left(x_{i j}\right)$ and $Y$ be matrices. Then $X \otimes Y$ denotes the matrix whose $(i, j)$ block is $x_{i j} Y$. If $(0) \leq V_{1} \leq V_{2} \leq V$ be $G F(2) H$ modules, then $V_{2} / V_{1}$ is a constituent of $V$. If $V_{2} / V_{1}$ is irreducible it is an irreducible constituent of $V$.

Lemma 1 Let $A$ be an elementary abelian 2-group, and let $V=V_{1} \oplus \cdots \oplus$ $V_{s}$ be a $G F(2) A$ module. Assume that $[V, A, A]=0$, and that $A$ permutes transitively on $\left\{V_{i} ; 1 \leq i \leq s\right\}$. Then $s \leq 2$, and if the equality holds, then $\left|A / C_{A}(V)\right|=2$. 
Proof. Assume that $s \geq 2$. Let $B$ be a maximal subgroup of $A$ which acts intransitively on $\left\{V_{i} ; 1 \leq i \leq s\right\}$. Since $\left(V_{1}^{B}\right)^{a} \cap V_{1}^{B}=0$ for $a \in A-B$ and $[V, A, A]=0$, it follows that $\left[V_{1}^{B}, B\right]=0$. This implies that $V_{1}^{B}=V_{1}$ and $s \leq 2$. Moreover, if the equality holds, then $[V, B]=\left[V_{1}^{A}, B\right]=\left[V_{1}, B\right]^{A}=0$ and $\left|A / C_{A}(V)\right|=2$.

Let $t$ be an integer. Let $S$ be a finite 2-group and $V$ be a $G F(2) S$ module. Then we write $V \in \mu_{t}(S)$ if $V=\left\langle v\right.$; $\left.\operatorname{dim}_{G F(2)}[v, A] \leq t\right\rangle$ for any elementary abelian subgroup $A$ of $S$ with $[V, A, A]=0$.

Lemma 2 Let $H$ be a finite group, and $T$ be a 2-subgroup of $H$. Let $V$ be a $G F(2) H$ module, and $U$ be a $T$ submodule of $V$ such that $U^{H}=V$ and $U \in \mu_{t}(T)$ for some inteder $t$. Let $A$ be an elementary abelian subgroup of $T$ with $[V, A, A]=0$. Then

(1) If $\left|A / A \cap O_{2}(H)\right| \leq 2^{n}$, then $V \in \mu_{t+n}(A)$.

(2) $U^{N_{H}(T)} \in \mu_{t}(T)$.

Proof. $\quad a \longmapsto[v, a]$ is a homomorphism on $A$ for each $v \in V$ and for $a \in A$. This implies (1). Since $U^{h} \in \mu_{t}(T)$ for all $h \in N_{H}(T),(2)$ follows from the definition.

Hypothesis A Let $p$ be an odd prime, $H$ be a finite $\{2, p\}$-group, $S \in$ $\operatorname{Syl}_{2}(H)$ and $P \in \operatorname{Syl}_{p}(H)$. Let $V$ be $a G F(2) H$ module and $U$ be an $S$ submodule of $V$ with $U^{H}=V$. Let $A$ be an elementary abelian subgroup of $S$ with $[V, A, A]=0$. Assume that $H=O_{2, p, 2}(H)$.

Lemma 3 Assume Hypothesis A. Further assume that

(a) $\mathrm{O}_{2}(H)=1$ and $P=[P, A]$; and

(b) $S=A$ and $|A| \geq 2^{2}$.

Then (1) There are generators $\left\{a_{i} ; i \in I\right\}$ of $A$ such that $\left[P, a_{i}\right] A<H$ for all $i \in I$.

Let $H_{i}=\left[P, a_{i}\right] A$ for $i \in I$. Then

(2) $V=\sum_{i \in I} U^{H_{i}}$.

Proof. (1) is obvious. To prove (2), let $W$ be an irreducible $H$ submodule of $V$ with $[W, P] \neq 0$ and let $s$ be the number of Wedderburn components of $W$ w.r.t. $P$. Let $\bar{H}=H / C_{H}(W)$. We will show that $|\bar{A}|=2$. By Lemma 1, $s \leq 2$, and if the equality holds, then $|\bar{A}|=2$. Assume that $s=1$. Then $\left|\Omega_{1} Z(\bar{P})\right|=p$ because $W$ is the sum of isomorphic $P$ submodules. Then 
we have that $1 \neq \Omega_{1} Z(\bar{P}) \leq \bigcap_{\bar{a} \in \bar{A}^{\sharp}}[\bar{P}, \bar{a}]$. By [1], we have that $|\bar{A}| \leq 2$. By $(\mathrm{a}),|\bar{A}|=2$. Thus we obtain $|\bar{A}|=2$. This implies that $\left[W, H_{i}\right] \neq 0$ if and only if $\left[W, a_{i}\right] \neq 0$, that is, $\left\langle\bar{a}_{i}\right\rangle=\bar{A}$ for $i \in I$. Let $I=\{1, \ldots, r\}$. Define the mapping $\rho$ from the set of all irreducible $H$ submodules $W$ of $V$ with $[W, P] \neq 0$ to $I$ as follows: $\rho(W)=i$ if and only if $\left[W, a_{j}\right]=0$ for all $j<i$ and $\left[W, a_{i}\right] \neq 0,1 \leq i \leq r$. Let $W_{i}$ be the sum of all irreducible $H$ submodules $W$ of $V$ with $\rho(W)=i, 1 \leq i \leq r$, and $W_{0}=C_{V}(P)$. Then we have that $V=\bigoplus_{i=0}^{r} W_{i}$. Let $\pi_{i}: V \rightarrow W_{i}$ be the natural projection, $0 \leq i \leq r$. Now we will show that

$$
\bigoplus_{j=0}^{i} W_{j} \leq \sum_{j=1}^{i} U^{H_{j}}, \quad 1 \leq i \leq r
$$

using induction on $i$. The assumption $V=U^{H}$ implies $W_{i}=\pi_{i}(U)^{H_{i}}$ for all $i$. Since

$$
\left[\bigoplus_{j=2}^{r} \pi_{j}(U), O^{2}\left(H_{1}\right)\right] \leq\left[\bigoplus_{j=2}^{r} W_{j}, O^{2}\left(H_{1}\right)\right]=0,
$$

we have that $W_{1} \leq U^{H_{1}}$. Assume that $\bigoplus_{j=0}^{i-1} W_{j} \leq \sum_{j=1}^{i-1} U^{H_{j}}$ for some $i \geq 2$. Since $\left[\bigoplus_{j=i+1}^{r} \pi_{j}(U), O^{2}\left(H_{i}\right)\right]=0$, it follows that

$$
W_{i} \leq \sum_{j=0}^{i} \pi_{j}(U)^{H_{j}} \leq \bigoplus_{j=0}^{i-1} W_{j}+U^{H_{i}} \leq \sum_{j=1}^{i} U^{H_{j}} .
$$

Therefore, $V=\bigoplus_{i=0}^{r} W_{i}=\sum_{i=1}^{r} U^{H_{i}}$, as required.

Lemma 4 Assume Hypothesis A. Let $\bar{H}=\mathrm{H} / \mathrm{O}_{2}(H)$. Further assume that

(a) $\bar{P}=[\bar{P}, \bar{A}]$; and

(b) $\bar{S}=\bar{A}$, and $|\bar{A}| \geq 2^{2}$.

Take $\left\{a_{i} ; i \in I\right\}$ so that $\left[\bar{P}, \bar{a}_{i}\right] \bar{A}<\bar{H}$ and let $H_{i}$ be the pre-image of $\bar{H}_{i}$ for $i \in I$. Then $V=\sum_{i \in I} U^{H_{i}}$.

Proof. We proceed using induction on $|U|$. Let $U_{0}$ be a maximal $S$ submodule of $U$, and $W=U_{0}^{H}$. By induction, $W=\sum_{i \in I} U_{0}^{H_{i}}$. It follows from the maximality of $U_{0}$ that $[(U+W) / W, S]=0$ and $\left[V / W, O_{2}(H)\right]=0$. By Lemma 3, we have that $V=\sum_{i \in I} U^{H_{i}}+W=\sum_{i \in I} U^{H_{i}}$. 
Theorem 1 Assume Hypothesis A. If $U \in \mu_{t}(S)$ for some integer t, then $V \in \mu_{t+1}(S)$.

Proof. We proceed using induction on $|H|$. Let $\bar{H}=H / O_{2}(H)$. It suffices to show that $V^{H} \in \mu_{t+1}(A)$. By way of a contradiction, assume that $V \notin$ $\mu_{t+1}(A)$. If $\bar{A}<\bar{S}$, then we have by induction that $V=U^{H}=U^{P}=$ $U^{O_{2}(H) A P} \in \mu_{t+1}(A)$, a contradiction. Hence, $\bar{S}=\bar{A}$. Let $R$ be the preimage of $[\bar{P}, \bar{A}]$. Assume that $R<P$. By the Frattini argument, $H=$ $R N_{H}(S)$. Since $U^{R} \in \mu_{t+1}(S)$ by induction, we have by Lemma 2 (2) that $V=U^{H}=\left(U^{R}\right)^{N_{H}(S)} \in \mu_{t+1}(S)$, a contradiction. Therefore, $\bar{P}=[\bar{P}, \bar{A}]$. By Lemma 2 (1), $|\bar{A}| \geq 2^{2}$. Then we can choose generators $\left\{a_{i}, i \in I\right\}$ of $A$ such that $\left[\bar{P}, \bar{a}_{i}\right] \bar{A}<\bar{H}$ forall $i \in I$. Let $H_{i}$ be the pre-image of $\left[\bar{P}, \bar{a}_{i}\right] \bar{A}, i \in I$. Now applying Lemma 4, we have by induction that $V=$ $U^{H}=\sum_{i \in I} U^{H_{i}} \in \mu_{t+1}(A)$, a contradiction. This completes the proof of the theorem.

Hypothesis B Let $H$ be a finite group, and $S \in \operatorname{Syl}_{2}(H)$. Let $V$ be a faithful $G F(2) H$ module. Let $\bar{H}=H / O_{2}(H)$. Let $\left\{\bar{K}_{i} ; 1 \leq i \leq r\right\}$ be the set of all components of $\bar{H}, E$ and $K_{i}$ be the pre-images of $E(\bar{H})$ and $\bar{K}_{i}$ respectively, $1 \leq i \leq r$. Let $U$ be an $S$ submodule of $V$ with $U^{H}=V$. Let $A$ be an elementary abelian subgroup of $S$ with $[V, A, A]=0$. Assume that $H=E S$.

If $O_{2}(H)=1$, we identify $\bar{X}$ with $X$ for a subset $X$ of $H$.

Lemma 5 Assume Hypothesis B. Let $L_{1}$ and $L_{2}$ be normal subgroup of $H$, enjoying the following properties:

(a) $L_{1} L_{2}=E$ and $\left[\bar{L}_{1}, \bar{L}_{2}\right]=1$; and

(b) Either $\left[V_{2} / V_{1}, L_{1}\right]=0$ or $\left[V_{2} / V_{1}, L_{2}\right]=0$ for any irreducible constituent $V_{2} / V_{1}$ of $V$.

Then $V=U^{L_{1}}+U^{L_{2}}$.

Proof. Assume first that $O_{2}(H)=1$. Let $W=C_{V}\left(L_{1}\right)+C_{V}\left(L_{2}\right)$. We may assume that $L_{1} \not \leq L_{2}$ and $L_{2} \not \leq L_{1}$. Then $O^{2}\left(L_{i}\right)=L_{i}, i=1,2$. Then [2, Theorem 3.15 p.187] implies that $C_{V / W}\left(L_{i}\right)=0, i=1,2$. By (b), $V / W=0$, that is, $V=C_{V}\left(L_{1}\right)+C_{V}\left(L_{2}\right)$. Let $V_{0}=C_{V}(E)$, and let $V_{i}$ be a complement submodule of $V_{0}$ in $C_{V}\left(L_{i}\right), i=1,2$. Let $u=\sum_{i=0}^{2} u_{i} \in U$, $h=h_{1} h_{2} \in E$, where $u_{i} \in V_{i}, 0 \leq i \leq 2$, and $h_{i} \in L_{i}, i=1,2$. Then it follows that $u^{h}=\sum_{i=0}^{2} u_{i}^{h_{1} h_{2}}=u_{0}+u_{1}{ }^{h_{2}}+u_{2}{ }^{h_{1}}=\left(u_{0}+u_{1}+u_{2}\right)+\left(u_{0}+\right.$ 
$\left.u_{1}+u_{2}^{h_{1}}\right)+\left(u_{0}+u_{1}^{h_{2}}+u_{2}\right)=u^{1}+u^{h_{1}}+u^{h_{2}} \in U^{L_{1}}+U^{L_{2}}$. Since $u$ and $h$ are arbitrary elements of $U$ and $E$ respectedly, we have that $V=U^{H}=U^{E}=$ $U^{L_{1}}+U^{L_{2}}$. Then we can handle the case $O_{2}(H) \neq 1$ by the similar way to the proof of Lemma 4.

Assume Hypothesis B. For $\bar{x} \in \bar{A}^{\sharp}$, define $\Theta(\bar{x})=\left\{i ;\left[\bar{K}_{i}, \bar{x}\right]=1,1 \leq\right.$ $i \leq r\}$. Define $\Theta(\bar{A})=\left\{\bar{x} \in \bar{A}^{\sharp} ; \Theta(\bar{x}) \neq \emptyset\right\}$. Note that if $\bar{x} \in \Theta(\bar{A})$, then $[\bar{E}, \bar{x}] \neq 1$ because $\bar{H}=\bar{E} \bar{S}$ and $\bar{x} \notin O_{2}(\bar{H})=1$.

Lemma 6 Assume Hypothesis B. Further assume that $V$ is irreducible, and that $\bar{H}=[\bar{E}, \bar{A}] \bar{A}$. Then $\Theta(\bar{A})=\emptyset$.

Proof. Note that $O_{2}(H)=1$, since $V$ is irreducible. By way of a contradiction, assume that $\Theta(\bar{A}) \neq \emptyset$. Let $x \in \Theta(\bar{A}), L_{1}=\left\langle K_{i} ; i \in \Theta(x)\right\rangle$ and $L_{2}=\left\langle K_{i} ; i \notin \Theta(x)\right\rangle$. Note that $L_{i}$ is $A$ invariant and normal in $H, i=1,2$ because $H=[E, A] A$. We may assume that $V$ has the only one Wedderburn component w.r.t. $E$. Otherwise, by Lemma $1|A|=2$ and $\Theta(\bar{A})=\emptyset$ because $[E, A]=E$. Then $V$ is the sum of isomorphic $E$ submodules. Let $F$ be a splitting field for $L_{1}$ and $L_{2}$ with characteristic 2 and $V^{*}=V \otimes F$. By [2, Theorem 3.7.1 and 2 pp. 100-102], $V^{*}$ is the sum of irreducible $E$ submodules of type $U_{1} \otimes U_{2}$, where $U_{i}$ is a faithful and irreducible $L_{i}$ module, $i=$ 1,2. Hence, either $\left(U_{1} \otimes U_{2}\right)^{h}=U_{1} \otimes U_{2}$ or $\left(U_{1} \otimes U_{2}\right)^{h} \cap U_{1} \otimes U_{2}=0$ for $h \epsilon$ $H$. Since $[E, A]=E$, we have that $\left[U_{1} \otimes U_{2}, A\right] \neq 0$. Then Lemma 1 implies $U_{1} \otimes U_{2}$ is $A$ invariant. Since $\left[L_{1}, x\right]=1$, we have that $\left[U_{1}, x\right]=0$. Let $X$ be the representation matrix of $x$ of the action on $U_{2}$. Since $[E, A]=E$, we have that $\left[L_{1}, A\right] \neq 1$. So, we can take $y \in A-C_{A}\left(L_{1}\right)$. Let $Y_{1}=\left(y_{i j}\right)$ and $Y_{2}$ be the representation matrices of $y$ of the actions on $U_{1}$ and $U_{2}$ respectedly. Then the representation matrices of $x$ and $y$ on $U_{1} \otimes U_{2}$ are $I \otimes X$ and $Y_{1} \otimes Y_{2}$ respectively, where $I$ is the identity matrix. Note that $[V, x, y]=0$ if and only if $(X-I)\left(Y_{2} y_{i i}-I\right)=O, 1 \leq i \leq n$, and $(X-I) Y_{2} y_{i j}=O$, $1 \leq i \neq j \leq n$, where $n=\operatorname{dim}_{F} U_{1}$ and $O$ is the zero matrix. Since $Y_{2}$ is non-singular, the latter equation yields $(X-I) y_{i j}=O$. Since $X \neq I$, we have that $y_{i j}=0,1 \leq i \neq j \leq n$. Therefore, $Y_{1}$ is a diagonal matrix. Then the former equation yields $y_{i i}(X-I)=(X-I) Y_{2}^{-1}=y_{j j}(X-I), 1 \leq i$, $j \leq n$. Since $X \neq I$, this implies that $Y_{1}$ is a scalar matrix. Since $L_{1}$ acts faithfully on $U_{1}$, it follows that $\left[L_{1}, y\right]=1$, contrary to the choice of $y$. This proves the assertion. 
Lemma 7 Assume Hypothesis B. Further assume that $\bar{H}=[\bar{E}, \bar{A}] \bar{A}$, and that $\Theta(\bar{A}) \neq \emptyset$. Let $\bar{x} \in \Theta(\bar{A}), \bar{L}_{1}=\left\langle\bar{K}_{i} ; i \in \Theta(\bar{x})\right\rangle$ and $\bar{L}_{2}=\left\langle\bar{K}_{i} ; i \notin\right.$ $\Theta(\bar{x})\rangle$. Let $L_{i}$ be the pre-image of $\bar{L}_{i}, i=1,2$. Then $V=U^{L_{1}}+U^{L_{2}}$.

Proof. Let $V_{2} / V_{1}$ be an irreducible constituent of $V$ and $C=C_{H}\left(V_{2} / V_{1}\right)$. Replacing $H$ by $H / C$ and $V$ by $V_{2} / V_{1}$ if necessary, we can apply Lemma 6 , and have that either $L_{1} \leq C$ or $L_{2} \leq C$. Then the assetion follows from Lemma 5.

Let $H$ be a finite group, let $L$ be a subgroup of $E(H)$ which is generated by some components of $H$. Let $V$ be an irreducible $G F(2) H$ module. Then we say that $V$ is a non-trivial product $L$ submodule, if $C_{V}(K)=0$ for all the components $K$ of $L$.

Lemma 8 Assume Hypothesis B. Further assume that $V$ is irreducible, and that $K_{1}^{A} \neq K_{1}$. Then $\left|A / N_{A}\left(K_{1}\right)\right|=2$. Moreover, one of the following holds:

(i) $\left[\bar{K}_{1}, N_{\bar{A}}\left(\bar{K}_{1}\right)\right]=1$; or

(ii) $\left[\bar{K}_{1}, N_{\bar{A}}\left(\bar{K}_{1}\right)\right]=\bar{K}_{1} \simeq S L\left(2,2^{n}\right)$ for some $n \geq 2,[\bar{E}, \bar{A}]=\bar{K}_{1}^{\bar{A}}$, and $V$ is a non-trivial product $\bar{K}_{1}^{\bar{A}}$ submodule.

Proof. Note that $O_{2}(H)=1$ because $V$ is irreducible. Throughout the proof, assume that (i) does not occur. Then $V$ has the only one Wedderburn component w.r.t. $E$, otherwise by Lemma $1|A|=2$ and (i) occurs, a contradiction. So, $V$ is the sum of isomorphic $E$ submodules. Thus, $C_{V}\left(K_{i}\right)=0,1 \leq i \leq r$. In paticular, $V$ is a non-trivial product $K_{1}^{A}$ submodule. Note that $V$ contains a faithful and irreducible $[E, A] A$ submodule. Let $F$ be a spltting field for all $K_{i}, 1 \leq i \leq r$, with characteristic 2 , and $V^{*}=V \otimes F$. By [2, Theorem 3.7.1 and 2], $V^{*}$ is the sum of irreducible $E$ submodules of type $\bigotimes_{i=1}^{r} U_{i}$, where $U_{i}$ is a faithful and irreducible $K_{i}$ module, $1 \leq i \leq r$. Since $\left[\bigotimes_{i=1}^{r} U_{i}, A\right] \neq 0$, Lemma 1 implies $\bigotimes_{i=1}^{r} U_{i}$ is $A$ invariant. Let $y \in A-N_{A}\left(K_{1}\right)$. Renumbering if necessary, we may assume that $U_{i}^{y}=U_{\ell+i}, 1 \leq i \leq \ell$ and $U_{i}^{y}=U_{i}, 2 \ell+1 \leq i \leq r$. Let

$$
W_{1}=\bigotimes_{i=1}^{\ell} U_{i} \text { and } W_{2}=\bigotimes_{i=\ell+1}^{2 \ell} U_{i} .
$$

Let $x \in N_{A}\left(K_{1}\right)-C_{A}\left(K_{1}\right)$. Note that $\left[K_{1}^{A}, x\right]=K_{1}^{A}$ because $A$ is abelian and $\left[K_{1}, x\right] \neq 1$ if and only if $\left[K_{1} / Z\left(K_{1}\right), x\right] \neq 1$. Assume that 
$\operatorname{dim}_{F} C_{W_{1}}(x) \geq 2$. Then we can choose $v_{1} \in C_{W_{1}}(x)-\{0\}$ and $v \in W_{1}$ such that $v+v^{x}$ is not contained in the subspace spanned by $v_{1}$. Let $v_{2}=$ $v+v^{x}$. Since $W_{1}^{y}=W_{2}$, we can choose bases $\left\{v_{i} ; 1 \leq i \leq t\right\}$ of $W_{1}$ and $\left\{w_{i} ; 1 \leq i \leq t\right\}$ of $W_{2}$ such that $v_{i}^{y}=w_{i}, 1 \leq i \leq t$. Note that $\left[v_{i}, x\right]=0$ if and only if $\left[w_{i}, x\right]=0,1 \leq i \leq t$. Let $w=v^{y}$. Then we have that $w^{x}+w=\left(v^{x}+v\right)^{y}=v_{2}^{y}=w_{2}$. It follows that

$$
\left[v \otimes w_{1}, y\right]=\left(v \otimes w_{1}\right)^{y}+v \otimes w_{1}=v_{1} \otimes w+v \otimes w_{1}
$$

and

$$
\begin{aligned}
{\left[v \otimes w_{1}, y, x\right] } & =\left(v_{1} \otimes w+v \otimes w_{1}\right)^{x}+\left(v_{1} \otimes w+v \otimes w_{1}\right) \\
& =v_{1} \otimes w^{x}+v^{x} \otimes w_{1}+\left(v_{1} \otimes w+v \otimes w_{1}\right) \\
& =v_{1} \otimes\left(w+w^{x}\right)+\left(v+v^{x}\right) \otimes w_{1}=v_{1} \otimes w_{2}+v_{2} \otimes w_{1} \neq 0 .
\end{aligned}
$$

This yields $\left[V^{*}, y, x\right] \neq 0$ and $[V, A, A] \neq 0$, a contradiction. Hence, $\operatorname{dim}_{F} C_{W_{1}}(x)=1$. Since $\operatorname{dim}_{F} W_{1}=\operatorname{dim}_{F} C_{W_{1}}(x)+\operatorname{dim}_{F}\left[W_{1}, x\right]$ and $\operatorname{dim}_{F}\left[W_{1}, x\right] \leq \operatorname{dim}_{F} C_{W_{1}}(x)=1$, we have that $\operatorname{dim}_{F} W_{1}=2$. Since $K_{1}$ is nonsolvable, we have that $\operatorname{dim}_{G F(2)} U_{1} \geq 2$. It must be that $W_{i}=U_{i}, i=$ 1,2 . This implies $K_{1} \simeq S L\left(2,2^{n}\right)$ for some $n \geq 2$. Moreover, $\left|A / N_{A}\left(K_{1}\right)\right|=$ 2 because $\ell=1$ and $y$ is an arbitrary element of $A-N_{A}\left(K_{1}\right)$. Now we will show that $[E, A]=K_{1}^{A}$. Assume that $E>K_{1}^{A}$. Let $W_{3}=\bigotimes_{i=3}^{r} U_{i}$. Let $X_{i}$ be the representation matrix of $x$ on $W_{i}, 1 \leq i \leq 3$. Let $Y_{1}$ and $Y_{2}$ be the representation matrices of $y$ on $W_{1} \otimes W_{2}$ and on $W_{3}$, respectedly. We may assume that $X_{1}=X_{2}=\left(\begin{array}{cc}1 & a \\ 0 & 1\end{array}\right)$ under bases $\left\{v_{i} ; 1 \leq i \leq 2\right\}$ and $\left\{w_{i} ; 1 \leq i \leq 2\right\}$ respectedly, where $a$ is a nonzero element of $F$, and $Y_{1}=\left(\begin{array}{cccc}1 & 0 & 0 & 0 \\ 0 & 0 & 1 & 0 \\ 0 & 1 & 0 & 0 \\ 0 & 0 & 0 & 1\end{array}\right)$ under a basis $\left\{v_{i} \otimes w_{j} ; 1 \leq i, j \leq 2\right\}$. Furthermore, we may set $X_{3}=\left(\begin{array}{cc}1 & A_{2} \\ O & A_{3}\end{array}\right)$ and $Y_{2}=\left(\begin{array}{cc}1 & B_{2} \\ O & B_{3}\end{array}\right)$ since the characteristic of $F$ is 2 . Let $X=X_{1} \otimes X_{2} \otimes X_{3}$ and $Y=Y_{1} \otimes Y_{2}$. Since $[V, x, y]=0$, we have that $(X-I)(Y-I)=O$. Then $(4,6)$ and $(5,4)$ blocks of $(X-$ $I)(Y-I)$ are $\left(A_{3}-I\right) B_{3}$ and $A_{2} B_{3}$ respectedly. Hence, $\left(A_{3}-I\right) B_{3}=O$ and $A_{2} B_{3}=O$. Since $B_{3}$ is non-singular, it follows that $A_{3}=I$ and $A_{2}=O$, that is, $X_{3}=I$ and $x$ acts trivially on $W_{3}$. Since $K_{i}$ acts faithfully on $W_{3}$ 
for $i \geq 3$, we have that $\left[K_{i}, x\right]=1$ for all $i \geq 3$. Hence, $[E, x]=K_{1}^{A}$. Since $V$ contains a faithful and irreducible $[E, A] A$ submodule, Lemma 6 implies that $\left[K_{i}, A\right]=1$ if and only if $\left[K_{i}, x\right]=1,1 \leq i \leq r$. Therefore, $[E, A]=[E, x]=K_{1}^{A}$.

Lemma 9 Assume Hypothesis B. Further assume that $K_{1}^{A} \neq K_{1}$, and that $\left[\bar{K}_{1}, N_{\bar{A}}\left(\bar{K}_{1}\right)\right]=\bar{K}_{1}$. Let $M_{1}=K_{1}^{A}$ and $M_{2}=\left\langle K_{i} ; \bar{K}_{i} \leq[\bar{E}, \bar{A}]\right.$ and $\left.K_{i} \not \subset K_{1}^{A}, 1 \leq i \leq r\right\rangle$ (possibly, $\left.M_{2}=O_{2}(H)\right)$. Then

(1) $\bar{K}_{1} \simeq S L\left(2,2^{n}\right)$ for some $n \geq 2$ and $\left|A / N_{A}\left(K_{1}\right)\right|=2$.

(2) Either $\left[V_{2} / V_{1}, M_{1}\right]=0$ or $\left[V_{2} / V_{1}, M_{2}\right]=0$ for any irreducible constituent $V_{2} / V_{1}$ of $V$.

(3) If $\bar{H}=[\bar{E}, \bar{A}] \bar{A}$ and $U \in \mu_{t}(S)$ for some integer $t$, then $V=$ $U^{M_{1}}+U^{M_{2}}$ and $U^{M_{1}} \in \mu_{t+n+1}(A)$. Moreover, if $U^{M_{1}} \notin \mu_{t+n}(A)$, then $\left|A / A \bigcap O_{2}\left(M_{1} A\right)\right|=2^{n+1}$.

Proof. Since $V$ is faithful, we can take an irreducible constituent $V_{2} / V_{1}$ of $V$ with $\left[V_{2} / V_{1}, K_{1}\right] \neq 0$. Let $C=C_{H}\left(V_{2} / V_{1}\right)$. By Lemma 8, $\left|A / N_{A}\left(K_{1}\right)\right|=$ 2 and $K_{1} / K_{1} \cap C \simeq S L\left(2,2^{n}\right)$ for some $n \geq 2$. Since $V_{2} / V_{1}$ is irreducible, we have $O_{2}(H) \leq K_{1} \cap C$. Since $O_{2}(\bar{H})=1, \bar{K}_{1} \cap \bar{C}$ is a subgroup of $Z\left(\bar{K}_{1}\right)$ of odd order. Since $K_{1} / K_{1} \cap C \simeq S L\left(2,2^{n}\right)$, we have $\bar{K}_{1} \cap \bar{C}=$ 1 and obtain (1). Moreover, Lemma 8 shows that $[E, A] C / C=K_{1}^{A} C / C$ and $M_{2} \leq C$. This implies (2) because $V_{2} / V_{1}$ is an arbitrary constituent of $V$ with $\left[V_{2} / V_{1}, K_{1}\right] \neq 0$. Assume that $\bar{H}=[\bar{E}, \bar{A}] \bar{A}$, and that $U \in$ $\mu_{t}(S)$. Then by (2) and Lemma 5, we have that $V=U^{M_{1}}+U^{M_{2}}$. Since $M_{1} / O_{2}\left(M_{1}\right) \simeq S L\left(2,2^{n}\right) \times S L\left(2,2^{n}\right)$ and $A \not \leq N_{H}\left(K_{1}\right)$, it is easy to see that $\left|A / A \cap O_{2}\left(M_{1} A\right)\right| \leq 2^{n+1}$. Then it follows from Lemma 2 $(1)$ that $U^{M_{1}} \in$ $\mu_{t+n+1}(S)$, and if $U^{M_{1}} \notin \mu_{t+n}(A)$, then $\left|A / A \bigcap O_{2}\left(M_{1} A\right)\right|=2^{n+1}$.

Theorem 2 Assume Hypothesis B. Let $m=\max _{1 \leq i \leq r}\left\{\log _{2} \mid N_{\bar{B}}\left(\bar{K}_{i}\right) /\right.$ $\left.C_{\bar{B}}\left(\bar{K}_{i}\right) \mid\right\}$, where $B$ ranges over all the subgroups of $S$ with $[V, B, B]=0$. If $U \in \mu_{t}(S)$ for some intger $t$, then one of the following holds:

(i) $V \in \mu_{t+m}(S)$; or

(ii) $V \in \mu_{t+m+1}(S)$, and there is a pair of an elementary abelian subgroup $A$ of $S$ and a component $\bar{K}_{i}$ of $\bar{H}$ such that $[V, A, A]=0$, $\left|A / N_{A}\left(K_{i}\right)\right|=2,\left|A / A \bigcap O_{2}\left(K_{i}^{A} A\right)\right|=2^{m+1}$ and $\bar{K}_{i} \simeq S L\left(2,2^{m}\right)$. Moreover, $V_{2} / V_{1}$ is a non-trivial product $\bar{K}_{i}^{\bar{A}}$ submodule and $[\bar{E}, \bar{A}]=$ $\bar{K}_{i}^{\bar{A}} C_{[\bar{E}, \bar{A}]}\left(V_{2} / V_{1}\right)$ for every irreducble constituent $V_{2} / V_{1}$ of $V$ with $\left[V_{2} / V_{1}, K_{i}\right] \neq 0$. 
Proof. Fix an elementary abelian subgroup $A$ of $S$ with $[V, A, A]=0$. Let $\Delta(A)$ be the set of all components $\bar{K}_{i}$ of $\bar{H}$ such that $K_{i}^{A} \neq K_{i}$, $\left|A / A \bigcap O_{2}\left(K_{i}^{A} A\right)\right|=2^{m+1}$ and $\bar{K}_{i} \simeq S L\left(2,2^{m}\right)$. Let $\ell=t+m$ if $\Delta(A)=\emptyset$ and $\ell=t+m+1$ if $\Delta(A) \neq \emptyset$. To prove $V \in \mu_{\ell}(S)$, we may only show that $V \in \mu_{\ell}(A)$ because $A$ is an arbitrary elementary abelian subgroup of $S$ with $[V, A, A]=0$. We will show that $V \in \mu_{\ell}(A)$ using induction on $|H|$. By way of a contradiction, assume that $V \notin \mu_{\ell}(A)$. If $E A<H$, then we have by induction that $V=U^{E}=U^{E A} \in \mu_{\ell}(A)$, a contradiction. Hence, $H=E A$. Let $L$ be the pre-image of $[\bar{E}, \bar{A}]$ and $T=L \bigcap S$. Since $L$ is normal in $H$, the Frattini argument shows that $H=L N_{H}(T)$. If $L A<H$, then we have by induction that $U^{L} \in \mu_{\ell}(T)$ and by Lemma 2 (2) that $V=\left(U^{L}\right)^{N_{H}(T)} \in$ $\mu_{\ell}(T) \subseteq \mu_{\ell}(A)$, a contradiction. Hence, $[\bar{E}, \bar{A}]=\bar{E}$. Assume that there is a component $\bar{K}_{i}$ of $\bar{H}$ such that $K_{i}^{A} \neq K_{i}$ and $\left[\bar{K}_{i}, N_{\bar{A}}\left(\bar{K}_{i}\right)\right]=\bar{K}_{i}$. Let $M_{1}=K_{1}^{A}$ and $M_{2}=\left\langle K_{i} ; K_{i} \not \leq K_{1}^{A}, 1 \leq i \leq r\right\rangle$. By Lemma 9, we have that $\bar{K}_{i} \simeq S L\left(2,2^{n}\right)$ for some $n \geq 2, V=U^{M_{1}}+U^{M_{2}}$ and $U^{M_{1}} \in \mu_{t+n+1}(A)$. Since $n \leq m$ and $M_{2} A<H$, we have that $V=U^{M_{1}}+U^{M_{2}} \in \mu_{\ell}(A)$, a contradiction. Therefore, we have that $N_{\bar{A}}\left(\bar{K}_{i}\right)=C_{\bar{A}}\left(\bar{K}_{i}\right)$, whenever $K_{i}^{A} \neq$ $K_{i}, 1 \leq i \leq r$. Now, we will show that $\Theta(\bar{A}) \neq \emptyset$. By Lemma 2 (1), we have that $|\bar{A}|>2^{\ell} \geq 2$. Assume that there is a component $\bar{K}_{i}$ of $\bar{H}$ such that $K_{i}^{A} \neq K_{i}$. By Lemma $9(1),\left|\bar{A} / C_{\bar{A}}\left(\bar{K}_{i}\right)\right|=\left|\bar{A} / N_{\bar{A}}\left(\bar{K}_{i}\right)\right|=2$. Since $|\bar{A}| \geq 2^{2}$, we have that $\Theta(\bar{A}) \neq \emptyset$. Assume next that $K_{i}$ is $A$ invariant for all $i$. Let $\pi_{i}$ be the natural homomorphism from $\bar{A}$ to $\bar{A} / C_{\bar{A}}\left(\bar{K}_{i}\right), 1 \leq$ $i \leq r$. If $\operatorname{Ker} \pi_{i}=1$ for all $i$, then it follows from the definition of $m$ that $|\bar{A}| \leq 2^{m}$, contrary to our assumption. Hence, $\operatorname{Ker} \pi_{i} \neq 1$ for some $i$ and $\Theta(\bar{A}) \neq \emptyset$. It is shown that $\Theta(\bar{A}) \neq \emptyset$. Let $\bar{x} \in \Theta(\bar{A}), \bar{L}_{1}=\left\langle\bar{K}_{i} ; i \in \Theta(\bar{x})\right\rangle$ and $\bar{L}_{2}=\left\langle\bar{K}_{i} ; i \notin \Theta(\bar{x})\right\rangle$. Let $L_{i}$ be the pre-image of $\bar{L}_{i}, i=1,2$. Now we can apply Lemma 7, and obtain that $V=U^{L_{1}}+U^{L_{2}}$. Since $L_{i} A<H$, $i=1,2$, we have that $U^{L_{i}} \in \mu_{\ell}(A), i=1,2$ and $V=U^{L_{1}}+U^{L_{2}} \in \mu_{\ell}(A)$, a contradiction. This proves $V \in \mu_{\ell}(A)$ and $V \in \mu_{\ell}(S)$. The remaining part of our assertion follows from Lemma 8 and 9. This completes the proof of the theorem.

Acknowledgement The author thanks to the refree for his helpful comments. 


\section{References}

[1] Alperin J.L., Quadratic fours groups. Communications in Algebra, 5 (7) (1977), 727-731.

[2] Gorenstein D., Finite Groups. Harper and Row 1968.

Department of Mathematics

Aichi University of Education

Kariya 448-8542, Japan

E-mai: thayashi@auecc.aichi-edu.ac.jp 